\title{
EINIGE BEMERKUNGEN ZUR MOLLUSKENFAUNA DES DOLLART-EMS-GEBIETES
}

\author{
S. VAN DER $\operatorname{HEIDE\bullet )}$
}

(mit 4 Tafeln)

\section{PROBENAHME}

Für die Mollusken war die Probeentnahme in den Prielen und Rinnen dieselbe wie für die Foraminiferen. Auf den Platen wurde jedoch an jeder Probeentnahmestelle einen, wenn möglich zwei Spaten tief gegraben und eine Mischprobe von 1 bis $2 \mathrm{dm}^{3}$ entnommen. Tiefere Ausgrabungen waren aus Zeitmangel nicht möglich. Es ist daher zu erwarten, daß die Zahlen der tiefer eingegrabenen Muscheln, vor allem von Mya arenaria (Linné) zu niedrig sind.

Die Proben wurden in einigen Fällen an Ort und
Stelle, meistens aber auf dem Schiff während der Rückfahrt gespült und in Alkohol aufbewahrt. Durch Färbung, wie dies bei den Foraminiferen beschrieben worden ist, wurde festgestellt, ob man es mit toten oder lebenden Exemplaren zu tun hatte.

Die Probeentnahme wurde größtenteils von den Assistenten A. VAn de Velde, F. Willemsen und G. SPAINK vorgenommen, während letzterer auch die Zählung der Muscheln durchführte. Diesen Herren sei hier für ihre unermüdliche Arbeit bestens gedankt.

\section{ALLGEMEINE BEMERKUNGEN ZUR MOLLUSKENFAUNA}

Das Dollart-Gebiet sowie das anstoßende Ems-Estuarium ist in Bezug auf seine Molluskenfauna ausgesprochen artenarm, während auch zahlenmäßig die Besiedelung mit Mollusken nicht stark ist. Nur Hydrobia ulvae (Pennant) und $\boldsymbol{H}$. stagnorum (Gmelin) sind etwas häufiger vertreten.

Im eigentlichen Dollart-Gebiet ist die Molluskenfauna fast ganz beschränkt auf die Arten: Hydrobia ulvae (Pennant), H. stagnorum (Gmelin), Macoma balthica (Linné) und Mya arenaria (Linné), nur spärlich begleitet von Scrobicularia plana (Da Costa), Cardium edule Linné und stellenweise Mytilus edulis Linné. Im Gebiet zwischen dem "Landpunt van Reide“ und dem südlichen Teil der „Oude Westereems" kommen einige wenige Arten wie Littorina littorea (Linné), Retusa alba (Kanmacher), Mysella bidentata (Montagu), Petricola pholadiformis Lamarck und Barnea candida (Linné) hinzu. Von diesen Arten kommt im Dollart-Gebiet nur Retusa alba (Kanmacher) ab und $\mathrm{zu}$ lebend vor, vereinzelte tote Exemplare von Littorina, Petricola und Barnea wur-

•) Geologischer Dienst, Haarlem. den dort ausnahmsweise angetroffen. Erst im nördlicheren Teil der „Oude Westereems“ zeigte sich eine Zunahme der Arten. Es handelte sich aber, abgesehen von einem lebenden Exemplar von Cardium edule Linné, nur um tote Exemplare. Angetroffen wurden (Fundstelle 319):

Hydrobia ulvae (Pennant)

Mytilus edulis Linné

Macoma balthica (Linné)

Mya arenaria (Linné)

Mysella bidentata (Montagu)

Abra alba (Wood)

Barnea candida (Linné)

Cardium edule Linné

Angulus fabulus (Gmelin)

Saxicavella jeffreysi Winckworth

Donax vittatus (Da Costa)

Montacuta ferruginosa (Montagu)

Hiatella arctica (Linné).

Eine ähnliche Annäherung an die Fauna des offenen Wattenmeeres wurde an der Ostseite von Profil VIII beobachtet, wo bei einer geringeren Artenzahl mehr lebende Exemplare gefunden wurden. Dazu wurde 
dort, von Süden kommend, zum ersten Mal Spisula subtruncata (Da Costa) angetroffen, jedoch nur in toten Exemplaren. Eine zwar tote Faunengesellschaft, welche repräsentativ für Nordsee-Wattenmeer ist, wurde an Fundstelle 328, westlich von Rottumeroog angetroffen. Neben den obengenannten Arten fanden sich:

Littorina littorea (Linné)

Retusa alba (Kanmacher)

Ostrea edulis Linné

Scrobicularia plana (Da Costa)

Spisula subtruncata (Da Costa)

Tellina tenuis Da Costa

(es fehlten hier Saxicavella, Montacuta und Hiatella). Zwischen diesen Faunen bildet die Molluskenfauna von Fundstelle 317 in der „Oude Westercems” eine merkwürdige Ausnahme. Sie enthält nicht weniger als 23 Arten. Viele Exemplare zeigen aber einen andern Erhaltungszustand als die normalen Faunenelemente dieser Gegend. Fügt man hinzu, daß diese Fauna 24 Exemplare von Bittium reticulatum (Da Costa) enthält, so ist es klar, daß es sich um eine Einspülung von Eemfossilien handelt. Als mehr oder veniger dieser Eemfauna angehörend betrachten wir:
Hydrobia ulvae (Pennant)

Littorina littorea (Linné)

Bittium reticulatum (Da Costa)

Turbonilla crenata (Brown)

Nassarius reticulatus (Linné)

Nucula nucleus (Linné)

Venerupis aurea senescens (Cocconi)

Scrobicularia plana (Da Costa)

Spisula subtruncata (Da Costa)

Aloidis gibba (Olivi)

Zirfaea crispata (Linné).

Dieses Auftreten von Eemfossilien an dieser Stelle ist bemerkenswert, weil im östlichen Teil der Provinz Groningen bis jetzt keine marinen Eemablagerungen im Untergrund angetroffen wurden. Daraus geht hervor, daß die Eemküste sich stärker ostwärts gebogen hat als man bis jetzt angenommen hat (Burck 1951, SpainK 1958). Es ist weiter auffallend, da $B$ an anderen Stellen keine Eemfossilien gefunden worden sind, abgesehen von einigen vereinzelten Exemplaren an Fundstelle 377, südlich von Rottumeroog. Der Transport von Mollusken scheint in diesem Gebiet sehr gering zu sein. Die Verbreitung von Hydrobia scheint diese Tatsache $\mathrm{zu}$ bestätigen (s. unten).

\section{DIE VERBREITUNG DER MOLLUSKEN IN DEN PRIELEN UND AUF DEN PLATEN}

Zunächst fällt auf, daß Hydrobia häufiger am Rande der Platen angetroffen wird, wenn dieser wenigstens nicht frisch abgetragen ist, Macoma balthica (Linné) und Mya arenaria (Linné) hingegen mehr auf den Platen selber, eine Verteilung, welche in Wattgebieten allgemein bekannt ist. Wir hatten im Dollartgebiet oft den Eindruck, daß die Besiedelung mit Macoma und Mya in der Mitte der Platen weniger stark war (z.B. in Fig. 1: Abnehmen von Macoma auf der Hering Plate, sowie von Mya auf der Kerkeriet Plate). Dies mag, besonders für Mya, auf einer Täuschung beruhen, weil man dort bei tieferem Ausgraben vielleicht eine größere Anzahl Exemplare gefunden hätte. Ist ja aus anderen Untersuchungen bekannt, daß langsam wühlende Arten, wie Mya, die ruhigen, also oberen Teile des Watts bevorzugen (ReIneck 1956). Ubrigens sind die Zahlen von Macoma und Mya im allgemeinen zu gering, um daraus Folgerungen ziehen zu können. Wir haben sie dennoch in Fig. 1-3 aufgezeichnet, um eine Ubersicht der Beobachtungen zu geben.

Bei der Verbreitung von Hydrobia spielt die Beschaffenheit des Bodens vielleicht eine etwas größere Rolle, dennoch scheint eine einfache Beziehung zwischen der physikalischen Bodenbeschaffenheit und der Hydrobia-Besiedelung nicht ohne weiteres zu bestehen. In Fig. 4 wurde die Zahl der lebenden Hydrobia-Exemplare angegeben, zusammen mit einer Kurve, welche den Prozentsatz des feinstkörnigen Materials darstellt. Diese Kurve zeigt in Profil IX eine zu geringe Variation, um als Beweis für die allerdings einigermaßen anwesende Ubereinstimmung gelten zu können. In Profil V zeigt nur Probe Nr. 277 eine gute Úbereinstimmung. Eine gewisse Beziehung mag da wirklich vorhanden sein. Gibt doch diese Probe gerade die Höchstzahl der lebenden HydrobiaExemplare in diesem Profil. In Profil III scheint ein gerader Gegensatz zwischen den beiden Kurven zu bestehen. Vermutlich wäre auch hier mehr Úbereinstimmung zu finden, wenn Korngrösse-Analysen aller Proben, insbesondere der Proben Nr. 195, Nr. 191 A und vielleicht Nr. 187, vorhanden wären. Das Fehlen einer allgemeinen Übereinstimmung macht es aber zweifelhaft, ob es angebracht wäre, dafür besondere Analysen zu machen.

Es ist fernerhin bemerkenswert, $\mathrm{daB}$ in Profilen, die bis an die Küste heranreichen (z.B. Profil III und IX) das Maximum von Hydrobia ganz nahe an der Küste liegt. Thamdrup (p. 108) hat dieses maximale Auftreten von Hydrobia ,besonders in den am läng- 
Hydrobia ulvae (Pennant)

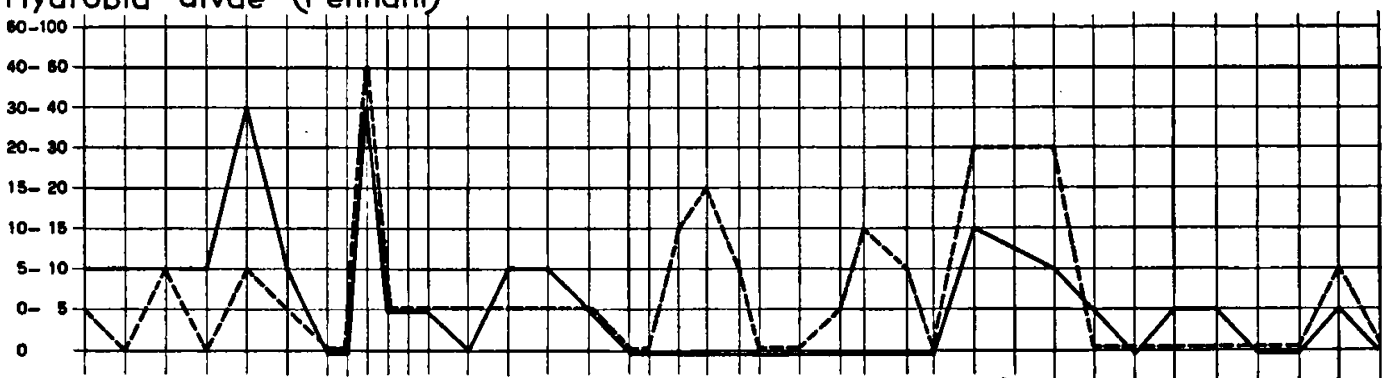

Hydrobia stagnorum (Gmelin) (Mir eventuell H. ulvae forma I)

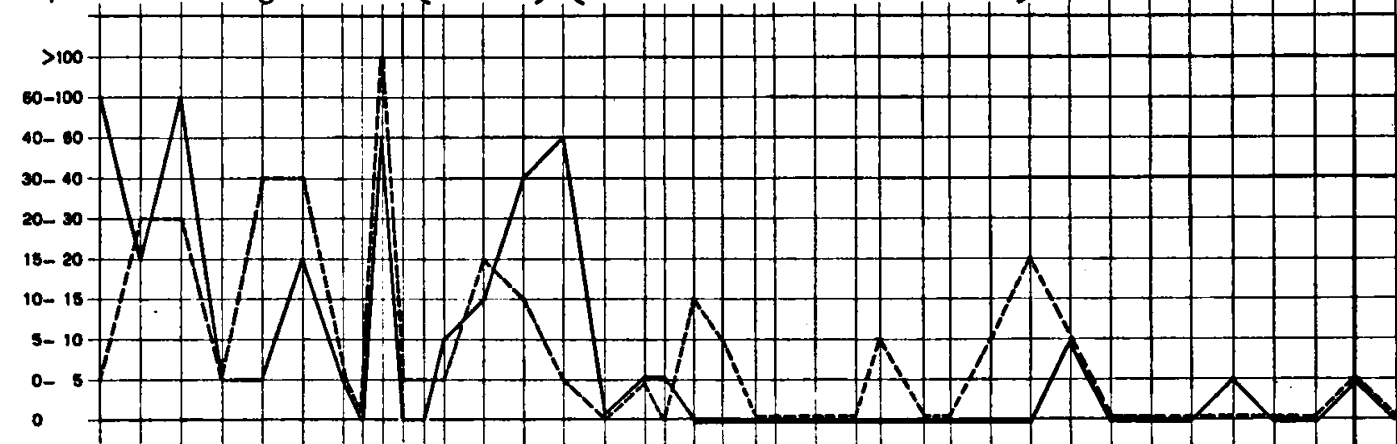

Macoma balthica (Linné)

60-100

40- 60

$30-40$

20- 30

15- 20

10- 15

5- 10

0- 8

0

Mya arenaria (Linné)
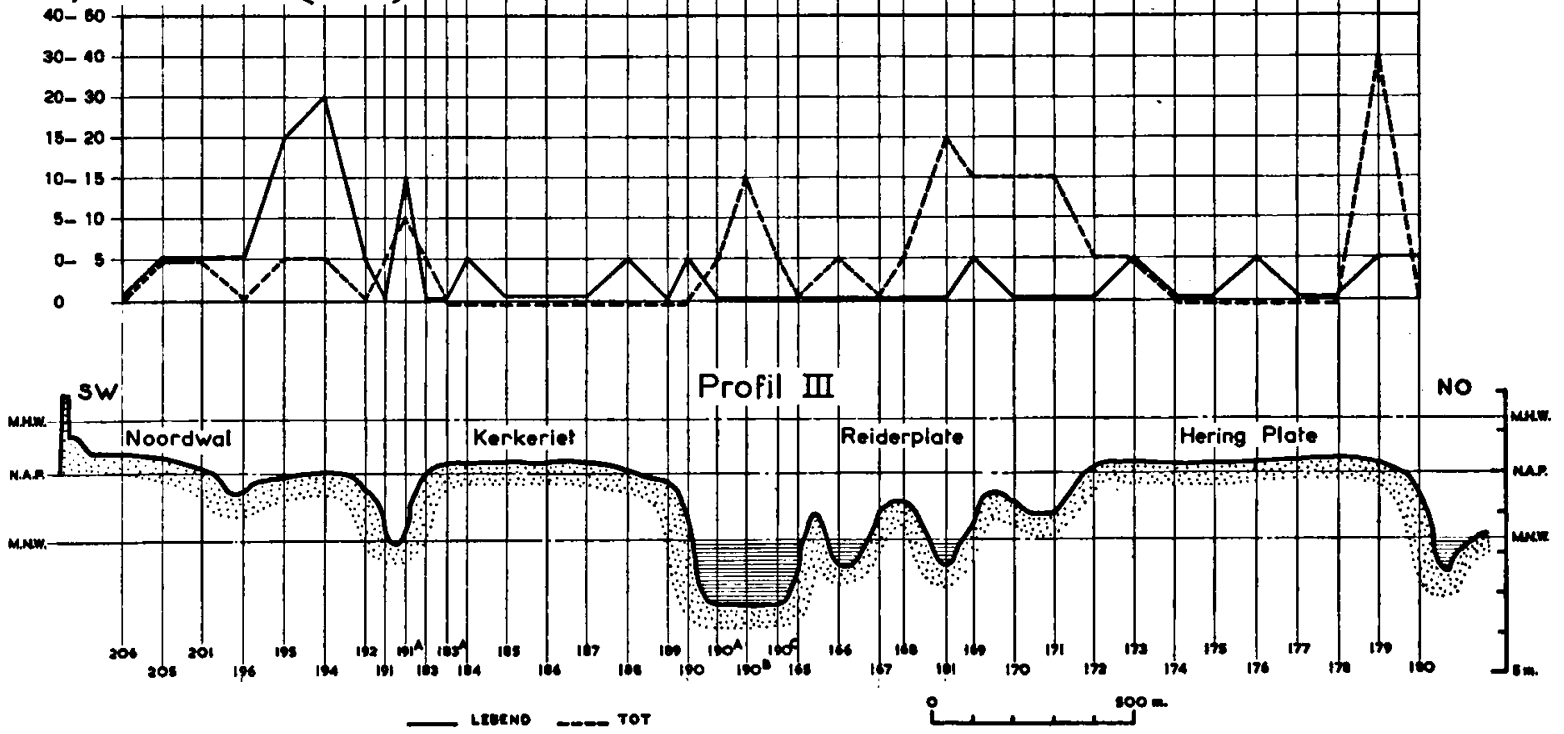

Fig. 1 


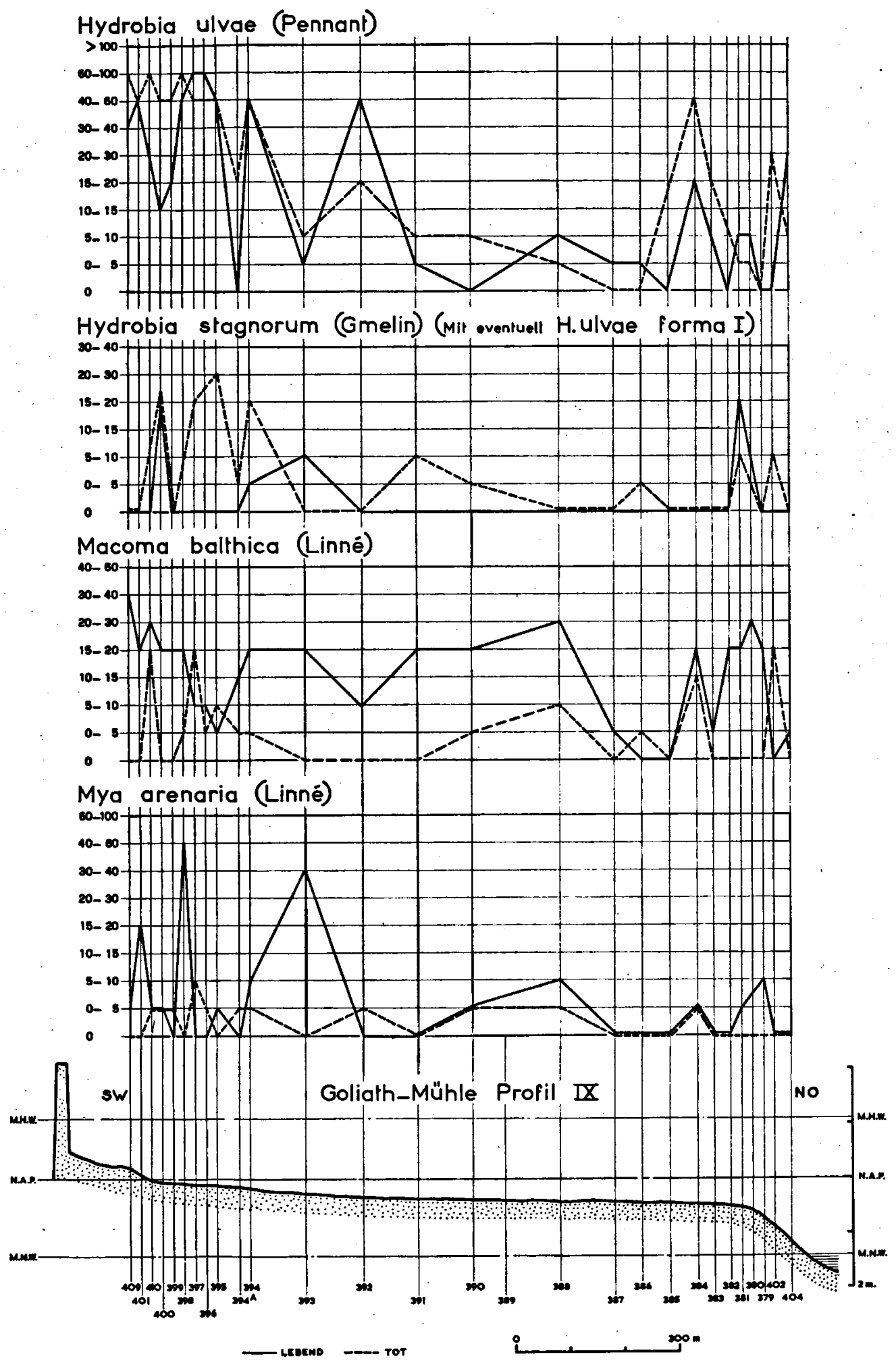

Fig. 2 


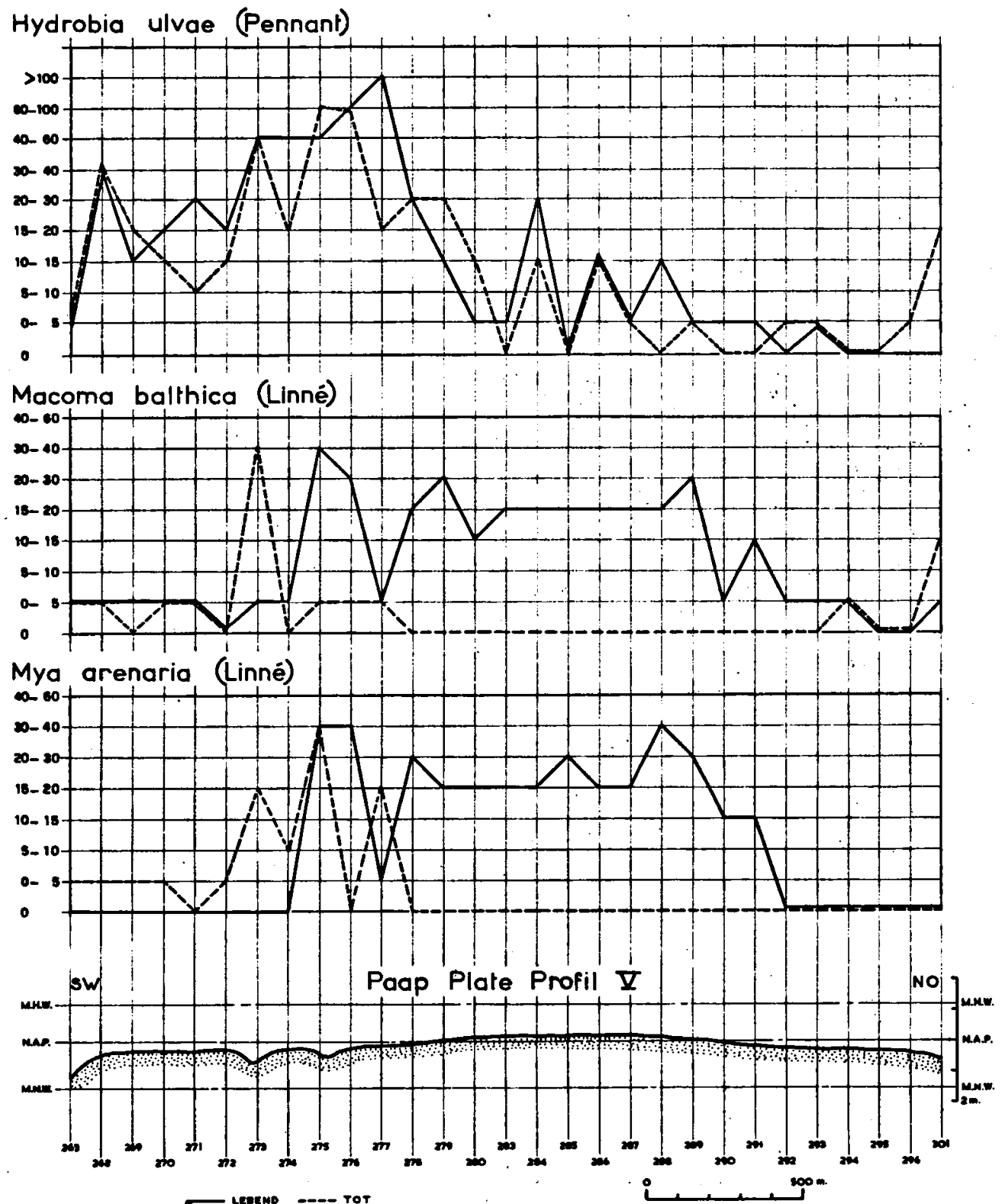

Fig. 3

sten trockenliegenden Teilen des Wattes" auch erwähnt. Er gibt dafür keine Erklärung. Es ist deutlich, $\mathrm{da} B$ unsere während kürzerer Zeit durchgeführten Beobachtungen keine SchluBfolgerungen erlauben.

In Fig. 1 sieht man weiter, daß lebende Mollusken in den Prielen fast völlig fehlen, eine im DollartEems-Gebiet allgemeine Erscheinung, welche mehr oder weniger einer Zunahme der Zahl toter Exemplare parallel läuft. Nur an zwei Stellen wurde eine
Ausnahme von dieser Regel gefunden, die weiter unten besprochen wird.

Fig. 1 zeigt an der Ostseite des Profils eine $\mathrm{Zu}$ nahme toter Exemplaren von Macoma und Mya. Hydrobia ist an diesem Prielrande weniger vertreten, die echte ulvae-Form weist sogar mehr tote als lebende Exemplare auf. Man hat hier mit einem in Erosion befindlichen Prielrande zu tun. Absterben der bloßgelegten Mya's ist in einem solchen Fall normal, die 

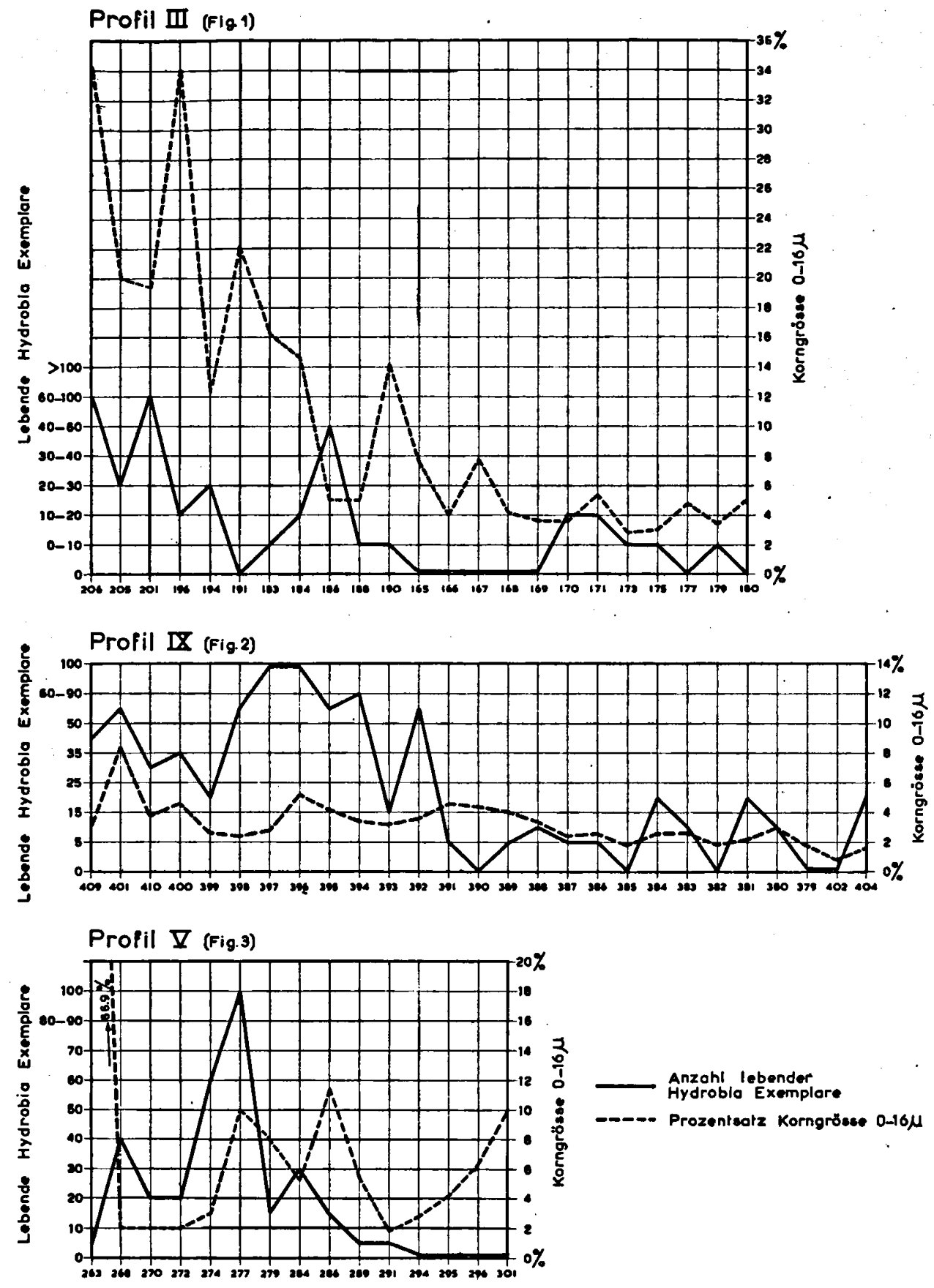

Fig. 4

Zunahme der toten Exemplaren von Macoma soll andere Ursachen haben (Bodenbeschaffenheit oder zu tief einschneidende Erosion), weil Macoma sonst allgemein imstande ist, sich wiedèr schnell einzugraben.

Wie oben erwähnt ist, wurden an zwei Stellen lebende Mollusken in Prielen gefunden. Erstens wurden an den Fundstellen 243 und 31233 bzw. 12 lebende Hydrobia-Exemplare angetroffen. KorngrößeVerteilung dieser Proben ist leider nicht vorhanden, es könnte aber sein, da $\beta$ hier ein sehr schlickiges Sediment anwesend ist (vgl. den außerordentlich 
hohen Gehalt an feinstkörnigem Material an Fundstelle 263). Das zweite Beispiel findet sich im eigentlichen Dollart-Gebiet in dem Priel an der Südseite der Hoogzand Plate. Die Verteilung der Anzahl lebender und toter Hydrobia-Exemplare ist dort:

\begin{tabular}{crrrrr} 
Fundstelle & 262 & \multicolumn{2}{c}{1} & leb. Ex. & \multicolumn{2}{c}{4 tote Ex. } \\
, & 261 & 4 & " & 4 & " \\
$"$ & 260 & 5 & $\#$ & 20 & $"$ \\
, & 259 & 19 & $\#$ & 59 &
\end{tabular}

Die Anzahl der Exemplare nimmt also nach der Prielmündung zu. Dabei ist die Zunahme bei den toten Exemplaren viel stärker. Eine nähere Betrachtung der Proben zeigt, daß sie außer den schon erwähnten Hydrobia-Exemplaren und abgesehen von ganz wenigen toten Schalen oder Fragmenten anderer Arten fast nur einige juvenile Exemplare (1 bis 3 lebende, 1 bis 9 tote Ex.) von Mya und Macoma enthalten. Es fragt sich, ob dieses lokale Auftreten lebender Mollusken in einem Priele nicht von einer anfangenden Verlagerung des Prieles herrührt. Sind doch die Ränder des Prieles oft von Hydrobia besiedelt, so daß bei anfangender Verlagerung diese $H y d r o b i a ' s$ abtransportiert werden, wobei zu erwarten ist, daß viele zugrundegehen. Mit dieser Vermutung stimmt überein, daß nur juvenile Exemplare von Mya und Macoma vorgefunden wurden, denn eine anfängliche Erosion kann eben die tieferen Schichten, worin sich die erwachsenen Exemplare befinden, noch nicht erreichen. Eine Bestätigung einer solchen Deutung wäre aber erwünscht und könnte nur durch regelmäßige, längere Zeit währende Beobachtungen gefunden werden.

Wenn Profil III (Fig. 1) ein repräsentatives Bild der Verbreitung der Mollusken im Dollart-Gebiet gibt, so muß auf eine merkwürdige Ausnahme hingewiesen werden, welche sich im Nordosten des Dollarts zeigte. Hier enthielt die Probeentnahmestelle 236 in Profil IV überhaupt keine Mollusken. Eine Deutung dieses völligen Fehlens von Mollusken ist schwer zu geben, es mag jedoch mit der Lage des Profils zwischen dem Flußgebiet der Ems und dem eigentlichen Dollart-Gebiet zusammenhängen.

Ein etwas anderes Bild zeigt Profil V (Fig. 3). Da findet man am südlichen Rande der Paap Plate eine normale Häufigkeit von Hydrobia. Die Nordseite dieser Plate ist aber sehr arm an Hydrobia. Hier spielt die Wasserbewegung und der Sedimenttransport eine deutliche Rolle, denn obgleich der Prozentsatz feinkörniger Teile von Fundstelle 291 nach 301 ansteigt (Fig. 4), gibt andrerseits Fundstelle 301 einen auffallenden Höchstwert für die Prozentsätze zwischen 150 und $300 \mu(39.9 \%)$. Das weist daraufhin, daß man tatsächlich mit der exponierten Seite der Plate zu tun hat. Demgegenüber zeigt Fundstelle 263 an der Südseite den Höchstwert für den Prozentsatz zwischen 0 und $16 \mu(69.9 \%)$.

\section{DIE VERBREITUNG DER HYDROBIA ULVAE FORMA I}

Es tritt im Dollart-Gebiet neben der gewöhnlichen Form von Hydrobia ulvae (Pennant) regelmäßig eine andere Form auf, die sich mit allen möglichen Zwischenstufen der Form von Hydrobia stagnorum (Gmelin) nähert. LiNKE (p. 293) erwähnt diese Form aus dem Jadebusenwatt: „Formen ..., die einen ziemlich gewölbten letzten Umgang, eine tief eingesenkte Naht und einen nach oben zu stumpf an den vorletzten Schalenumgang anstoßenden Mündungsrand haben, Merkmale, die für $\boldsymbol{H}$. stagnalis (Baster) bezeichnend sind". Wie LiNKE weiter erwähnt ist ein sicheres Auseinanderhalten nur möglich, wenn man die Radula zu Hilfe nimmt. Vorläufig haben wir diese Form mit dem Namen Hydrobia ulvae forma I angedeutet. Neben dieser Form tritt im DollartGebiet auch die echte Form von Hydrobia stagnorum (Gmelin) auf.

Deutliche Formen von Hydrobia stagnorum (Gmelin) sind z.B. anwesend im westlichsten Teil von Profil III. Eine ähnliche Häufigkeit landwärts (bzw. wattwärts) zeigt Profil IV, wo die Verbreitung von Nord nach Süd folgende ist:

\begin{tabular}{|c|c|c|}
\hline Fundstelle & 227 & 4 lebende Ex. \\
\hline 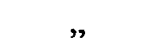 & 232 & 6 lebende Ex. \\
\hline e. & 233 & 27 lebende Ex. \\
\hline & 234 & 18 lebende und 12 tote Ex. \\
\hline$"$ & 235 & 16 lebende und 31 tote Ex. \\
\hline " & 236 & keine Ex. \\
\hline$"$ & $\begin{array}{l}237 \\
238\end{array}$ & $\begin{array}{l}\text { keine Ex. } \\
20 \text { lebende und } 32 \text { tote Ex. }\end{array}$ \\
\hline$"$ & $\begin{array}{l}239 \\
240\end{array}$ & $\begin{array}{l}54 \text { lebende Ex. } \\
69 \text { lebende Ex. }\end{array}$ \\
\hline
\end{tabular}

Mit diesem Vorkommen hängt wahrscheinlich die Anwesenheit eines toten Exemplars in der Ems (Fundstelle 241) und eines lebenden und 7 toter Exemplare weiter flußabwärts (Fundstelle 242) zusammen.

Es ist aber auffallend, daß die typische stagnorumForm, mit Ausnahme der obenerwähnten Exemplare 
in der Ems, außerhalb des eigentlichen Dollart-Gebietes an keiner Fundstelle angetroffen wurde.

Hydrobia ulvae forma I fehlt vom Dollart-Gebiet nordwärts in fast allen Proben. Nur Fundstelle 345 (Profil VII) weist ein lebendes und zwei tote Exemplare dieser Form auf. An dieser Stelle ist die Entfernung vom Dollart-Gebiet aber noch nicht besonders groß. Im übrigen Gebiet, das heißt in der „Bocht van Watum“, auf den Platen Paap und Hond, wurde diese Form kein einziges Mal angetroffen. Sie tritt erst wieder auf in Profil IX. Dort enthalten 6 Proben vereinzelte lebende und tote Exemplare, weitere 6 enthalten nur einige tote Exemplare. Häufiger wird sie erst wieder in Profil VIII (südlich von Rottumeroog), wo ziemlich viele lebende Exemplare und einige tote Exemplare an Fundstelle 355 vorgefunden wurden. Drei andere Fundstellen dieses Profils enthielten vereinzelte tote Exemplare, nur Fundstelle 363 lieferte einige lebende Exemplare dazu.

Das Interessante an dieser Verbreitung ist das
Fehlen von Hydrobia stagnorum (Gmelin) außerhalb des Dollart-Gebietes und das Fehlen von Hydrobia ulvae forma $I$ in großen Teilen des Ems-Estuariums. Gegenüber dem massenhaften Auftreten dieser letzten Form im zentralen Dollart-Gebiet ist es bezeichnend, daß keine Exemplare (auch keine toten) in den gut beobachteten Gegenden von Hund und Paap vorgefunden wurden. Das Vorkommen vereinzelter Exemplare, unter denen lebende, an der Küste in Profil VII und IX deutet mehr darauf hin. daß diese Form in geringer Zahl im küstennahen Gebiet lebt, als daß sie dorthin verfrachtet wurde. Südlich von Rottumeroog (Profil VIII) hat man offensichtlich (bei der großen Zahl lebender Exemplare an Fundstelle 355) mit einer echten Lebensgemeinschaft zu tun.

Man möchte aus diesen Beobachtungen die Folgerung ziehen, $\mathrm{da} \beta$ Transport von Mollusken über größere Entfernungen im Dollart-Eemsgebiet fast nicht stattfindet. Das lokale Auftreten der Eemfossilien deutet in derselben Richtung.

\section{ZUSAMMENFASSUNG}

Die Molluskenfauna des Dollart-Ems-Gebietes, besonders des eigentlichen Dollart-Gebietes, ist artenarm. Eine Anreicherung der Molluskenfauna in der „Oude Westereems“ deutet auf örtliche Vermischung mit Eemfossilien hin. Im allgemeinen ist Hydrobia stärker vertreten an den Rändern der Priele. Sie hat ihr maximales Auftreten im höchstgelegenen Teil des Watts. Die Besiedelung der Platen besteht hauptsächlich aus Macoma balthica (Linné), Mya arenaria (Linné), und in bedeutend geringerem Masse Scrobicularia plana (Da Costa).
Abgesehen von zwei örtlichen Ausnahmen wurden in den Prielen nur tote Exemplare vorgefunden. Eine dieser Ausnahmen wäre vielleicht durch anfangende seitliche Erosion des Prieles zu erklären. Ubrigens weist das nur sehr örtliche Vorkommen von Eemfossilien, sowie die Verbreitung der Hydrobia-Formen, auf einen im allgemeinen geringen Transport im ganzen Dollart-Ems-Gebiet hin.

Die Mollusken sind aufbewahrt im Zoologischen Museum in Amsterdam.

\section{SCHRIFTTUM}

ANKeL, W. E. (1929) - Hydrobienschill und Hydrobienkalk. Natur und Museum, Bd. 59, p. 33.

BENTHEM JUTting, T. van (1922) - Zoet- en brakwatermollusken. In: Flora en Fauna der Zuiderzee.

Burck, H. D. M. (1951) - Het continentale RiB-Würm interglaciaal. Geol. en Mijnb., N.S., $13^{\circ}$ Jg., p. 290.

LINKE, O. (1939) - Die Biota des Jadebusenwattes. Helgol. Wiss. Meeresunters., Bd. 1, p. 201.

ReinecK, H. E. (1956) - Der Wattenboden und das
Leben im Wattenboden. Natur und Volk, Bd. 86, p. 268.

SPAINK, G. (1958) - De Nederlandse Eemlagen I. Wetens. Meded. Kon. Ned. Natuurhist. Ver., no. 29.

Thamdrup, H. M. (1935) - Beiträge zur Okologie der Wattenfauna. Medd. Skalling-Laboratoriet, Bd. II.

WoHLENBERG, E. (1937) - Die Wattenmeer-Lebensgemeinschaften im Königshafen von Sylt. Helgol. Wiss. Meeresunters., Bd. 1, p. 1. 